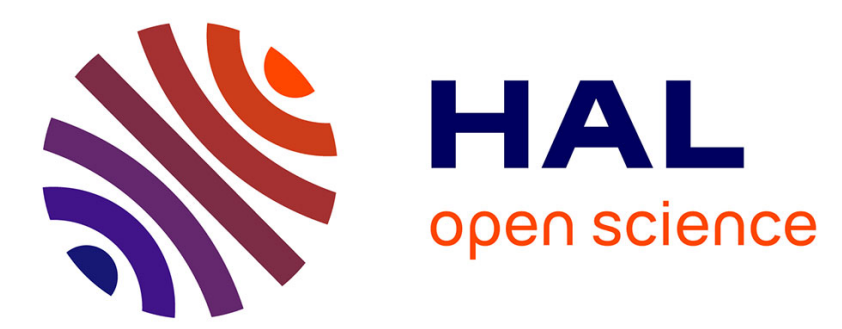

\title{
Hybrid dynamic modeling and control of switched affine systems: application to DC-DC converters
}

\author{
Carolina Albea-Sanchez, Germain Garcia, Luca Zaccarian
}

\section{To cite this version:}

Carolina Albea-Sanchez, Germain Garcia, Luca Zaccarian. Hybrid dynamic modeling and control of switched affine systems: application to DC-DC converters. 54th IEEE Conference on Decision and Control, Dec 2015, Osaka, Japan. hal-01220447v3

\section{HAL Id: hal-01220447 \\ https://hal.science/hal-01220447v3}

Submitted on 14 Mar 2017

HAL is a multi-disciplinary open access archive for the deposit and dissemination of scientific research documents, whether they are published or not. The documents may come from teaching and research institutions in France or abroad, or from public or private research centers.
L'archive ouverte pluridisciplinaire HAL, est destinée au dépôt et à la diffusion de documents scientifiques de niveau recherche, publiés ou non, émanant des établissements d'enseignement et de recherche français ou étrangers, des laboratoires publics ou privés. 


\title{
Hybrid dynamic modeling and control of switched affine systems: application to DC-DC converters
}

\author{
Carolina Albea, Germain Garcia and Luca Zaccarian
}

\begin{abstract}
The paper deals with the problem of control of switched systems described by a set of affine differential equations. Among the potential applications, the DC-DC converters constitute an important class of systems, which concentrates the interest of the control community. The paper proposes a new formulation of the problem in the context of hybrid dynamic systems, which represents an adequate way for handling the requirements of DC-DC converters, while guaranteeing theoretically and practically all specifications in terms of stability and performance. In this sense, the proposed approach encompasses several methods considered in the literature. The method is illustrated for the cases of buck and boost converters. The developed results are preliminary but constitute an interesting direction for reducing the gap between theoretical results and their practical applications in power electronics.
\end{abstract}

\section{INTRODUCTION}

The recent developments in power electronics are justified by the necessary conversion of electric energy in the domain of industrial and domestic applications. These last decades, significant advances have been done in several connected domains like for example, electronic components, materials, control methods, CAD tools... which allow for the design of highly reliable and efficient power processing systems [13]. Consequently, the need for power processing systems characterized by high frequency, reliability and low cost has increased drastically. In the context of low current and voltage levels, the high frequency switching converters play a fundamental role and are involved in a lot of applications such as transport systems, informatics and telecommunications to cite few of them. This explains why, these last years, in conjunction with the developments evoked above, the control of DC-DC power converters has been revisited in the light of these achievements. DC-DC power converters are switched systems that can be regarded in several ways. The discontinuous nature associated with their operation, generates a dynamical complexity, not easy to handle rigorously with standard mathematical tools. However, in several practical cases, a DC-DC converter operates around an equilibrium point, it is possible to

C. Albea is with both CNRS, LAAS, 7 avenue du colonel Roche, F-31400 Toulouse, France and Univ. de Toulouse, UPS, LAAS, F31400 Toulouse France calbea@laas.fr

G. Garcia is with both CNRS, LAAS, 7 avenue du colonel Roche, F-31400 Toulouse, France and Univ. de Toulouse, INSA, LAAS, F31400 Toulouse France garcia@laas.fr

L. Zaccarian is with CNRS, LAAS, 7 avenue du colonel Roche, F-31400 Toulouse, Univ. de Toulouse, LAAS, F-31400 Toulouse, France and Dipartimento di Ingegneria Industriale, University of Trento, Italy zaccaian@laas.fr derive averaged models, in general bilinear, allowing the use of all the methods developed in the context of nonlinear systems (feedback linearization, flatness, passivity... see for example [12] and references therein) or, in the case of linear models obtained from linearization of bilinear averaged ones, the use of powerful linear control design techniques (see for example, [2], [9], [14]). Among the main limitations, we can point out a difficulty for quantifying the precision of the approximation obtained by averaging procedure and the fact that the control laws properties are only valid locally.

More recently, the control community has concentrated some efforts to the study of new hybrid control techniques [7] such as the switched systems, a subclass of hybrid systems characterized by a switching rule which selects, at each instant of time, a mode in a set of possible modes, each of them corresponding to a dynamic subsystem [3], [5]. Applications to the case of DC-DC converters can be found for example in [1] where the problem is formulated in terms of control of a switched system whose modes are described by affine differential equations. However, it is possible to show that the obtained switching rules can be interpreted as sliding mode control laws with sliding surfaces implicitly determined in terms of the state space variables (currents, voltages) and of the selected equilibrium operation [8]. Even if the implicit discontinuous nature of DC-DC converters is taken into account by an appropriate switched model, the limitation is due to the practical implementation of the control law whose properties are only ensured for an infinite switching frequency, while for practical reasons, the switching frequency must be constrained.

The objective of this paper is to go a step further and to state a control problem in the context of the control of hybrid dynamic systems [4] which seems to be an adequate way for handling the specificities of DC-DC converters while guaranteeing theoretically and practically, that the implemented control laws will satisfy all the specifications in terms of stability and performance. The interest is the possibility of identifying and managing some design parameters affecting important practical indicators such as: switching frequency, dissipated energy, overshoot... which are not easy to manage for methods like sliding mode control for example. In this paper, the considered paradigm is the one developed in [4] which associates to a continuous-time flow whose model is of a differential equation type, a discrete-time behavior capturing the switched characteristic imposed by the control law and necessary for energy conversion operations. Two subsets included in an extended space, 
built from the state and the control spaces, determine the regions where the continuous and discrete dynamics are active. The main tool for proving stability of a compact attractor defined in this extended space, proceeds by an appropriate extension of Lyapunov stability theory developed in the context of hybrid dynamic systems in [4] and [10]. Due to the affine structure of the modes, a quadratic Lyapunov function can be selected from a positive definite symmetric matrix satisfying a set of Lyapunov inequalities. A hybrid control law with its two associated flow and jump is deduced from this matrix and an upper bound of a LQ performance index for the controlled system can be computed. It is possible to deduce an optimal guaranteed cost control law leading to the tight upper bound, by solving a LMI optimization problem. Although the results proposed in this paper must be considered as preliminary, they are promising and could allow solving generic control design problems encountered in the domain of power electronics.

The paper is organized as follows. The next section states the problem, defines the considered switched affine models and gives the adopted assumptions. Section II proposes the hybrid dynamical model formulation with the corresponding control law. Section IV discusses optimality issues and Section $\mathrm{V}$ illustrates the proposed theory for two well-known DC-DC converters, namely the buck and the boost converters. A conclusion ends the paper.

\section{Problem formulation}

Inspired by the work in [1], we focus on the following class of switched affine systems, which is relevant in the context of DC-DC converters:

$$
\begin{aligned}
& \dot{x}=A_{u} x+B_{u} V_{i n}, \\
& z=C_{u} x,
\end{aligned}
$$

where the available input $u$ is the switching signal, assigning a specific desired logical mode among $N$ possible ones:

$$
u \in \mathbb{K}:=\{1,2, \ldots, N\} .
$$

Moreover, in dynamics (1), $V_{i n} \in \mathbb{R}^{m}$, is a constant voltage source, $x \in \mathbb{R}^{n}$ is the state, $z \in \mathbb{R}^{p}$ is the controlled output, and $A_{u}$ and $B_{u}$ present suited dimensions.

This paper focuses on the design problem of a feedback law for the switching signal $u$, in such a way to ensure suitable convergence properties of the plant state $x$ to a value $x_{e}$, which is not necessarily an equilibrium for the continuous-time dynamics in (1), but can be obtained as an equilibrium for the switching system with arbitrary switching. A necessary and sufficient condition characterizing this equilibrium is then represented by the following standard assumption (see [1], [6]).

Assumption 1: There exists $\lambda_{e}=\left[\lambda_{e_{1}}, \lambda_{e_{2}}, \ldots, \lambda_{e_{N}}\right]$ satisfying $\sum_{l=1}^{N} \lambda_{e_{l}}=1$, such that the following convex combination holds:

$$
\sum_{l=1}^{N} \lambda_{e_{l}}\left(A_{l} x_{e}+B_{l} V_{i n}\right)=0
$$

Remark 1: It is emphasized that Assumption 1 is both necessary and sufficient for the existence of a suitable switching signal ensuring forward invariance of the point $x_{e}$ (namely inducing an equilibrium at $x_{e}$ ) when understanding solutions in the generalized sense of Krasovskii or Filippov. Indeed, under (3), this signal is a periodic sequence of arbitrary small period $T$, spending a time equal to $\lambda_{e_{i}} T$ in mode $i$. Conversely, if Assumption 1 does not hold, such a signal does not exist because any arbitrary switching signal can only generate an equivalent action on $\dot{x}$ corresponding to a convex combination of the right hand sides obtained with each mode (namely, equation (3)).

In the next section we will propose a hybrid formalism for the design problem associated to finding a switching signal $u$ suitably stabilizing a point $x_{e}$ satisfying Assumption 1. To this end, mimicking the conditions already presented in [1], we will assume the existence of the following set of matrices $P$ and $Q_{i}, i \in \mathbb{K}$.

Property 1: Given matrices $A_{i}, i \in \mathbb{K}$ in (1), there exists a matrix $P=P^{T}>0 \in \mathbb{R}^{n \times n}$ and matrices $Q_{i}=Q_{i}^{T}>0 \in \mathbb{R}^{n \times n}, i \in \mathbb{K}$, satisfying

$$
A_{i}^{T} P+P A_{i}+2 Q_{i}<0
$$

for all $i \in \mathbb{K}$.

Note that Property 1 enforces the strong requirement that all matrices $A_{i}$ be Hurwitz. Suitable relaxations of (4) are possible and correspond to only imposing a similar bound for a convex combination of matrices $A_{i}$. We save these relaxations as future work and concentrate on this simpler case in this paper.

Selection of matrices $P$ and $Q_{i}, i \in \mathbb{K}$ satisfying Property 1 will be discussed in Section IV where an optimization-based procedure will be suggested, also in light of the stability and optimality theorems presented below.

\section{HYBRID MODEL AND PROPOSED CONTROL LAW}

We formulate here the design problem as a hybrid dynamical system, following the formalism in [4], wherein continuous-time behavior resembles the evolution in (1), and the discrete-time behavior captures the jump of the control signal $u$ from one mode to another. We represent the overall dynamics as:

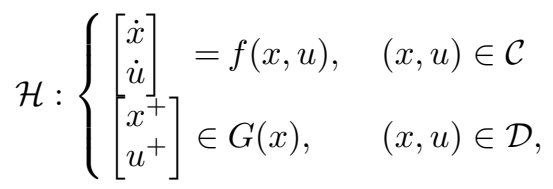

where $G$ is a (set-valued) map capturing the switching logic:

$$
\begin{aligned}
& f(x, u):=\left[\begin{array}{c}
A_{u} x+B_{u} V_{i n} \\
0
\end{array}\right] \\
& G(x):=\left[\begin{array}{c}
x \\
\underset{i \in \mathbb{K}}{\operatorname{argmin}}\left(x-x_{e}\right)^{T} P\left(A_{i} x+B_{i} V_{i n}\right)
\end{array}\right]
\end{aligned}
$$

and where the so-called "flow" and "jump" sets $\mathcal{C}$ and $\mathcal{D}$ encompass, respectively, the regions in the (extended) 
space $(x, u)$ where our switching strategy continues with the current mode $u$ (set $\mathcal{C}$ ) or is required to switch to a new mode (set $\mathcal{D}$ ). If switching is allowed (namely, if $(x, u) \in \mathcal{D})$ then $u$ will switch according to $G$ in $(6)$. For the solution proposed in this paper, we select the flow and jump sets based on the desired equilibrium $x_{e}$ introduced in Assumption 1 and on the parameters $P$ and $Q_{i}, i \in \mathbb{K}$ introduced in Property 1 as follows:

$$
\begin{aligned}
\mathcal{C} & :=\left\{(x, u): \tilde{x}^{T} P\left(A_{u} x+B_{u} V_{i n}\right) \leq-\eta \tilde{x}^{T} Q_{u} \tilde{x}\right\} \\
\mathcal{D} & :=\left\{(x, u): \tilde{x}^{T} P\left(A_{u} x+B_{u} V_{i n}\right) \geq-\eta \tilde{x}^{T} Q_{u} \tilde{x}\right\},
\end{aligned}
$$

where $\tilde{x}=x-x_{e}$ and scalar $\eta \in(0,1)$ is a design parameter that will be shown to be useful for suitably achieving a trade-off between switching frequency and optimality level as characterized later in Theorem 2 and commented in Remark 3.

Remark 2: Note that hybrid system (5)-(8) provides a solution strategy for the selection of the switching signal $u$. Indeed, sets (7) and (8) correspond to a specification about "when" a jump is or is not allowed, and map $G$ in (6) specifies where a solution "may" jump (that is, what values of $u$ are allowed after the jump. It is emphasized that both these elements of our solution may be sources of non-uniqueness of the solutions. Indeed, sets in (7) and (8) have overlapping boundaries so that multiple solutions (flowing or jumping) may arise from the same initial condition. Similarly, the argmin in the definition of $G$ in (6) may be nonunique. Despite this non-uniqueness feature, the stability and optimality results proven below refer to all possible solutions and therefore it is not really important what solutions we select in a possible implementation of this control law (or in a MATLAB simulation) because stability and optimality properties hold for all of them.

The next lemma is a fundamental step to prove our main result in Theorem 1 below.

Lemma 1: Consider matrices $P \in \mathbb{R}^{n \times n}$ and $Q_{i} \in$ $\mathbb{R}^{n \times n}, i \in \mathbb{K}$ satisfying Property 1 , a point $x_{e} \in \mathbb{R}^{n}$ satisfying Assumption 1. Then, for each $x \in \mathbb{R}^{n}$,

$$
\min _{i \in \mathbb{K}} \tilde{x}^{T} P\left(A_{i} x+B_{i} V_{i n}\right) \leq \min _{i \in \mathbb{K}}-\tilde{x}^{T} Q_{i} \tilde{x} .
$$

While the proof of Lemma 1 is given in Section VI, we comment here on its relevance in terms of the nature of the switching signals generated by our solution. In particular, property (9) combined with (7) shows that unless $\tilde{x}=\tilde{x}^{+}=0$ (which means that we are at the equilibrium $x=x_{e}$ ), the solution always jumps to the interior of the flow set $\mathcal{C}$. Indeed, $\tilde{x} \neq 0$ implies

$$
-\tilde{x}^{T} Q_{u} \tilde{x}<-\eta \tilde{x}^{T} Q_{u} \tilde{x},
$$

because $\eta<1$. This fact, together with stability (ensuring boundedness of solutions) and the sector growth condition coming from the linearity of the flow dynamics (1), implies that there is a uniform lower bound on the dwell time between each pair of consecutive resets before solutions approach $x=x_{e}$. Clearly this lower bound shrinks to zero as solutions approach $x=x_{e}$ because only arbitrarily fast switching can make $x_{e}$ and equilibrium, in general. As compared to existing approaches (see e.g., [1]) that essentially rely on a "sliding-mode" type of paradigm, thereby typically leading to very fast switching and chattering along a sliding surface, our solution is instead characterized by relatively slow switching, where the switching frequency can be adjusted, to a certain extent, using parameter $\eta$ (see Remark 3 below for extra details).

Following up to standard stability theory for hybrid systems [4], we will establish suitable stability properties of the point $x_{e}$ in terms of uniform global attractivity of a bounded (and closed) set in the higher-dimensional space spanned by $(x, u)$. In particular, we will establish properties of the following compact attractor:

$$
\mathcal{A}:=\left\{(x, u): x=x_{e}, u \in \mathbb{K}\right\},
$$

encompassing the fact that we are interested in uniform stability and convergence to a set where $x=x_{e}$ and $u$ assumed some unspecified value or pattern within the desired limit set of solutions. The following theorem, whose proof is given in Section VI, is the main result of our paper.

Theorem 1: Consider a point $x_{e}$ satisfying Assumption 1 and matrices $P \in \mathbb{R}^{n \times n}$ and $Q_{i} \in \mathbb{R}^{n \times n}, i \in \mathbb{K}$ satisfying Property 1 . Then attractor (10) is uniformly globally asymptotically stable (UGAS) for hybrid system (5)-(8). Moreover, UGAS is robust because the attractor (10) is compact.

\section{OPtimality AND PARAMETERS TUNING}

Theorem (1) establishes UGAS of the attractor, which results in desirable uniform stability and convergence properties. However, we are interested in further providing a suitable performance guarantee for our solution, which follows the same paradigm as that one in [1]. This performance guarantee, may for example refer to desirable levels of dissipated energy, current peak, response time among others.

Within the considered hybrid context, we first recall that solutions are parametrized by ordinary time $t$ (measuring amount of flow) and discrete-time $j$ (measuring the number of switches) so that the domain of a solution $\xi$ (see [4, Ch. 2]) corresponds to a finite or infinite union of intervals of the following form:

$$
\operatorname{dom} \xi=\bigcup_{j \in \operatorname{dom}_{j} \xi} I^{j} \times\{j\}
$$

with $I^{j}=\left[t_{j}, t_{j+1}\right]$ being a bounded time interval having the so-called "jump times" $t_{k}$ as extremes, or possibly being a last unbounded interval open to the right and of the form $I^{j}=\left[t_{j},+\infty\right)$. In (11), we use the notation $\operatorname{dom}_{j} \xi:=\{j \in \mathbb{Z}:(t, j) \in \operatorname{dom} \xi$, for some $t \in$ $\mathbb{R}\}$, namely $\operatorname{dom}_{j} \xi$ includes all $j \in \mathbb{Z}$ such that $I^{j}$ is non-empty. Within this context, we represent an LQ performance metric focusing on flowing characteristics of the plant state, using the following expression:

$$
J(\xi):=\sum_{k \in \operatorname{dom}_{j}} \int_{t_{k}}^{t_{k+1}}|\tilde{z}(\tau, k)|^{2} d \tau
$$


where $\xi=(x, u): \operatorname{dom} \xi \rightarrow \mathbb{R}^{n} \times \mathbb{K}$ is a solution to hybrid system $(5)-(8), \tilde{z}(t, j)=C_{u(t, j)}, \tilde{x}(t, j)$ for all $(t, j) \in$ $\operatorname{dom} \xi$.

With our hybrid switching solution, we may then give the following guarantee on the performance cost (12). The proof of the next statement is given in Section VI.

Theorem 2: Consider hybrid system (5)-(8) satisfying Assumption 1 and Property 1. If

$$
C_{i}^{T} C_{i} \leq Q_{i}, \quad \forall i \in \mathbb{K},
$$

then the following bound holds along any solution $\xi=$ $(x, u)$ of $(5)-(8)$ :

$$
J(\xi) \leq \eta^{-1} \tilde{x}(0,0)^{T} P \tilde{x}(0,0),
$$

where $\tilde{x}(t, j)=x(t, j)-x_{e}$, for all $(t, j) \in \operatorname{dom}(\xi)$.

Remark 3: It should be emphasized that once matrices $P$ and $Q_{i} \in \mathbb{R}^{n \times n}, i \in \mathbb{K}$ have been fixed compliantly with requirement (13), the guaranteed performance level for our scheme (in terms of size of the upper bound for index $J$ in (12) along solutions) is proportional to the inverse of $\eta \in(0,1)$ (see (14)). To this end, large values of $\eta$ (as close as possible to 1) are expected to lead to improved LQ performance along solutions.

On the other hand, one may appreciate by looking at the flow and jump sets in (7) and (8), that smaller values of $\eta$ correspond to strictly smaller jump sets (and larger flow sets), which reveals that solutions are expected to flow longer before switches of control input $u$ are experienced. Therefore we anticipate that solutions with smaller values of $\eta$ exhibit a smaller switching frequency. In other words, one may play with parameter $\eta$ to suitably adjust the switching frequency along solutions. This operation clearly affects the level of guaranteed optimality, according to (14).

The problem addressed next is the computation of parameters $P, Q_{i}, i \in \mathbb{K}$, following some kind of optimization with the goal of reducing as much as possible the right hand side in bound (14). To this end, we make the following natural selection of matrices $Q_{i}, i \in \mathbb{K}$ :

$$
Q_{i}=C_{i}^{T} C_{i}+\varepsilon I, \quad i \in \mathbb{K},
$$

where $\varepsilon>0$ is a (typically small) positive constant, which may be selected equal to zero if $C_{i}^{T} C_{i}>0$ for all $i \in \mathbb{K}$. Then it is clear that selection (15) ensures $Q_{i}>0, \forall i$, as required, in addition to ensuring bound (13).

Once parameters $Q_{i}$ are selected, under the assumption that $A_{i}$ are Hurwitz matrices for all $i \in \mathbb{K}$, the following convex optimization expressed by linear matrix inequalities always leads to a feasible solution:

$$
\begin{aligned}
\min _{P=P^{T}>0} & \text { Trace } P, \text { subject to: } \\
& A_{i}^{T} P+P A_{i}^{T} \leq-2 Q_{i}, \quad \forall i \in \mathbb{K},
\end{aligned}
$$

and this optimal solution clearly satisfies Property 1.

\section{Applichtion to CONVERTERs With two CONFIGURATIONS}

The hybrid control scheme given before is tested in two classic DC-DC converters and compared to the switched control proposed in [1]. The DC-DC converters are: a buck converter and a boost converter. These converters switch between $N=2$ affine subsystems, being the state variable $x=\left[\begin{array}{ll}i_{L} & v_{c}\end{array}\right]^{T}$, where $i_{L}$ denotes the inductor current and $v_{c}$ denotes the capacitor voltage. Given a desired equilibrium $x_{e}=\left[\begin{array}{ll}i_{e} & v_{e}\end{array}\right]^{T}$, we select the cost function $J$ in (12) as follows:

$\min _{u} \sum_{k \in \operatorname{dom}_{j}(\xi)} \int_{t_{k}}^{t_{k+1}} \frac{\rho}{R_{0}}\left(v_{c}(\tau, k)-v_{e}\right)^{2}+R\left(i_{L}(\tau, k)-i_{e}\right)^{2} d \tau$

where $\rho$ is a positive scalar. Note that the constant parameters of each term express the weighted sum of the energy of the error signal of each state variable.

Following (15), we take

$$
Q_{1}=Q_{2}=\left[\begin{array}{cc}
R & 0 \\
0 & \frac{\rho}{R_{0}}
\end{array}\right] .
$$

We take the parameters given in [1] for comparison with the switched control algorithm presented therein. The considered nominal values are: $V_{i n}=100 \mathrm{~V}, R=2 \Omega$, $L=500 \mu \mathrm{H}, C_{o}=470 \mu \mathrm{F}$ and $R_{o}=50 \Omega$. Moreover, we take a sampling time $T_{s}=10^{-6} s$ and $\rho=1000$ for all topologies. Note that, we give more weight to the voltage than to the current, expecting to obtain a voltage convergence faster at the expense of a larger current peak. Simulations are performed in MATLAB/Simulink by using the HyEQ Toolbox [11].

\section{A. Buck converter}

The switched system state space model (1) is defined by the following matrices:

$$
A_{1}=A_{2}=\left[\begin{array}{cc}
-R / L & -1 / L \\
1 / C & -1 / R_{0} C_{0}
\end{array}\right], B_{1}=\left[\begin{array}{c}
1 / L \\
0
\end{array}\right], B_{2}=\left[\begin{array}{l}
0 \\
0
\end{array}\right] .
$$

The simulation parameters chosen are:

$$
x_{e}=\left[\begin{array}{ll}
0.8 & 40
\end{array}\right]^{T}, P=\left[\begin{array}{ll}
0.28 & 0.47 \\
0.47 & 1.16
\end{array}\right] \cdot 10^{-2} .
$$

Note that Property 1 is satisfied. Figure 1 shows the voltage and current evolutions of the buck converter controlled by our proposed hybrid dynamic control scheme and by the switched control proposed in [1], for different values of $\eta$. Note that the voltage evolution is essentially the same, the transient lasts less than $1 \mathrm{~ms}$ at the expense of a current peak close to $15 \mathrm{~A}$ of magnitude. In this simulation, we stabilize the point corresponding to $\lambda_{e}=$ [0.43 0.57], satisfying Assumption 1. Thus, the buck converter with our hybrid control scheme guarantees UGAS of the attractor in (10) (from Theorem 1) and optimality guarantees arising from Theorem 2. These properties can be appreciated from the simulations of Fig. 1.

On the other hand, the current peak performance is more suited when $\eta$ is larger. For $\eta=0.99$, we obtain essentially the same output performance as the one of 
the switched control proposed in [1]. However, we insist that choosing such a large value of $\eta$ is not a good idea in terms of switching frequency. In particular, Fig. 2 shows the switching frequency in a time slot of the transient for different values of $\eta$. From the figure it appears that using $\eta=0.99$ we obtain the same frequency as the one obtained with the switched control proposed in [1] (lower two plots). Nevertheless, according to Remark 3 , as $\eta$ is reduced, a reduced switching frequency is expected, which is consistent with the upper two plots of Fig. 2. This trend of the switching frequency with respect to $\eta$ can be well appreciated in Fig. 3 that shows the normalized switching frequency and cost function $(J)$ as a function of $\eta$ (different curves correspond to different initial conditions). The curves are normalized to the switching frequency experienced with the method of [1]. As expected, for large values of $\eta$, we recover the same frequency as the one in [1], but reducing $\eta$ we may give up a little on optimality level and suitably adjust the switching frequency. In particular, for $\eta<0.5$ we start seeing some relevant and interesting reduction. We note that this behavior related to the value of $\eta$ can be exploited for on-line adjustment of the frequency through adaptation of $\eta$. We regard this research direction as future work.
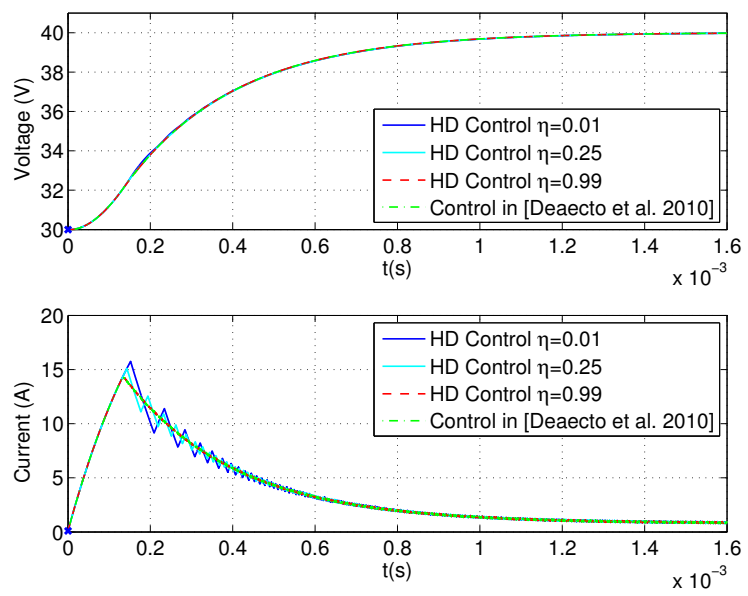

Fig. 1: Voltage and current evolution of the buck converter.

\section{B. Boost converter}

The switched system state space model (1) is defined by the following matrices:

$$
\begin{gathered}
A_{1}=\left[\begin{array}{cc}
-R / L & 0 \\
0 & -1 / R_{0} C_{0}
\end{array}\right], \quad A_{2}=\left[\begin{array}{cc}
-R / L & -1 / L \\
1 / C_{0} & -1 / R_{0} C_{0}
\end{array}\right], \\
B_{1}=B_{2}=\left[\begin{array}{c}
1 / L \\
0
\end{array}\right] .
\end{gathered}
$$

The chosen simulation parameters are:

$$
x_{e}=\left[\begin{array}{ll}
3 & 120
\end{array}\right]^{T}, P=\left[\begin{array}{ll}
1.45 & 0.09 \\
0.09 & 2.48
\end{array}\right] \cdot 10^{-2},
$$

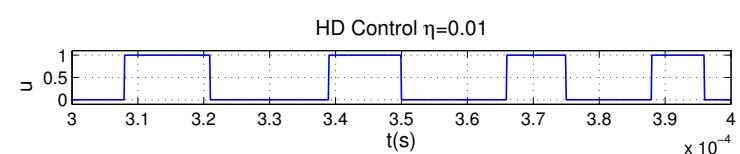

HD Control $\eta=0.25$
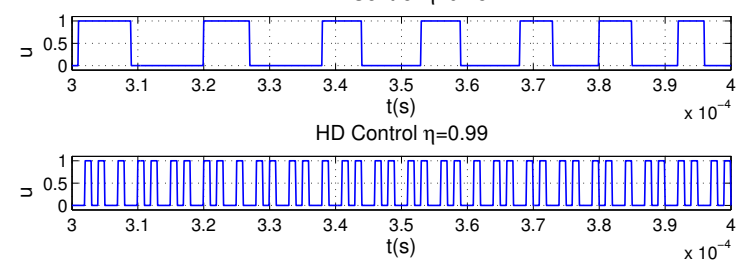

Control in [Deaecto et al. 2010]

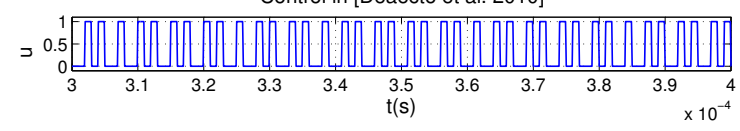

Fig. 2: Zoom of $u$ in the buck converter.

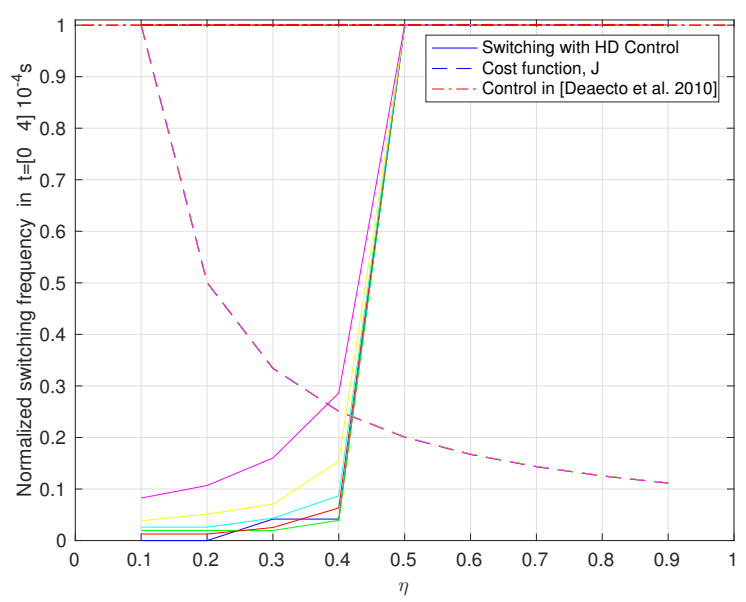

Fig. 3: Evolution of the normalized switching frequency and cost function, $J$, w.r.t. $\eta$ for different initial conditions in the buck converter.

which satisfy Property 1 (this is easily checked numerically). Similar to the buck converter case (and the corresponding Fig.1), Fig. 4 reports voltage and current evolutions for different selections of $\eta$ and for the choice $\lambda_{e}=\left[\begin{array}{ll}0.22 & 0.78\end{array}\right]$ that satisfies Assumption 1. Just as before, Theorems 1 and 2 guarantee asymptotic stability and optimality for the scheme. Once again, as $\eta$ gets larger, the transient becomes closer to the one obtained when the switched control proposed in [1]. In all cases, the transient duration is less than $30 \mathrm{~ms}$ with a maximum current peak near $3.25 \mathrm{~A}$ of magnitude. The switching frequency in a time slot of the transient is reported in Fig. 5 for different values of $\eta$. Similar to the previous Fig. 2, we see the expected trend (from Remark 3 ) of the switching frequency, as a function of $\eta$. A more informative picture can be grasped by Fig. 6 where, similar to the previous Fig. 3, it is shown that selecting small values of $\eta$ allows us to suitably adjust the switching frequency while giving up a little on the performance guarantee 
(even though Fig. 4 shows that the performance is not much deteriorated when $\eta$ is very small, thus showing some level of conservativeness of our bound). Note that, as compared to the previous case of Fig. 3, the value of $\eta$ for which we obtain essentially the same behavior as the scheme in [1] is much larger than 0.5 and grows up to somewhere around 0.95 .
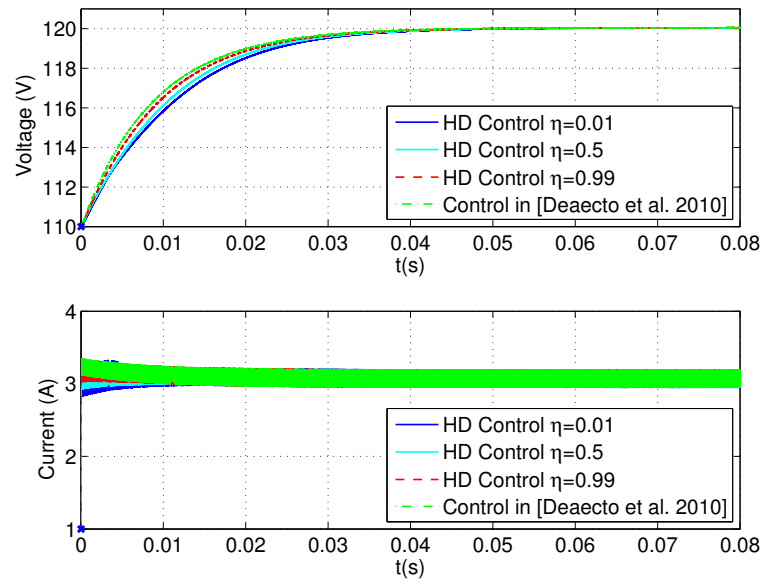

Fig. 4: Voltage and current evolution of the boost converter.
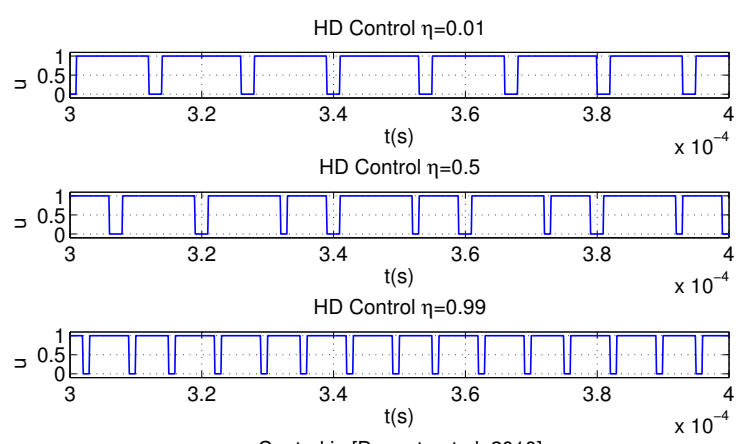

Control in [Deaecto et al. 2010]

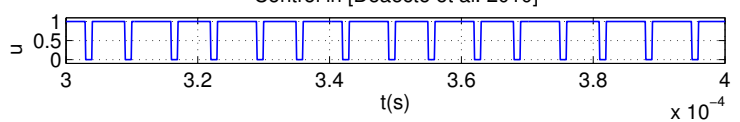

Fig. 5: Zoom of $u$ in the boost converter.

\section{Proof OF THE MAIN RESUlts}

Proof of Lemma 1. First notice that the left hand side of $(3)$ is linear in $\lambda_{n}=\left[\lambda_{1}, \lambda_{2}, \ldots, \lambda_{N}\right]$, and $\lambda_{e}$ allows to the compact set $\Lambda=$ $\left\{\lambda_{n}=\left[\lambda_{1}, \lambda_{2}, \ldots, \lambda_{N}\right], \lambda_{n} \geq 0, \sum_{l=1}^{N} \lambda_{n}=1\right\}$. Then, the following minimum is obtained at the extreme

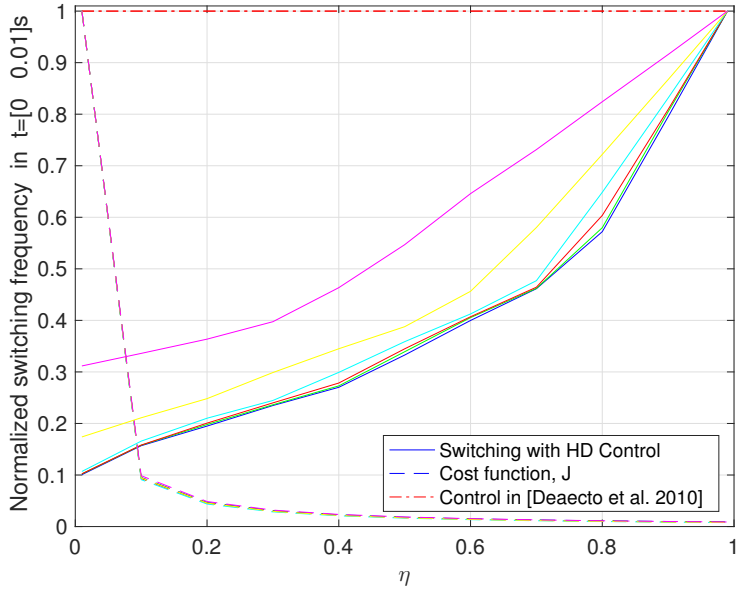

Fig. 6: Evolution of the normalized switching frequency and cost function, $J$, w.r.t. $\eta$ for different initial conditions in the boost converter.

points:

$$
\begin{aligned}
& \min _{i \in \mathbb{K}} \tilde{x}^{T} P\left(A_{i} x_{e}+B_{i} V_{i n}\right) \\
& =\min _{\lambda_{n} \in \Lambda} \tilde{x}^{T} P\left\{\sum_{l=1}^{N} \lambda_{n_{l}} A_{l} x_{e}+\sum_{l=1}^{N} \lambda_{n_{l}} B_{l} V_{i n}\right\} \\
& \leq \tilde{x}^{T} P\left\{\sum_{l=1}^{N} \lambda_{e_{l}} A_{l} x_{e}+\sum_{l=1}^{N} \lambda_{e_{l}} B_{l} V_{i n}\right\}=0 .
\end{aligned}
$$

Then, the proof easily follows from applying (4) and (17) as follows

$$
\begin{aligned}
& \min _{i \in \mathbb{K}} \tilde{x}^{T} P\left(A_{i} x+B_{i} V_{i n}\right) \\
& \leq \min _{i \in \mathbb{K}} \tilde{x}^{T} P A_{i} \tilde{x}+\min _{i \in \mathbb{K}}\left(\tilde{x}^{T} P A_{i} x_{e}+\tilde{x}^{T} P B_{i} V_{i n}\right) \\
& \leq \min _{i \in \mathbb{K}}-\tilde{x}^{T} Q_{i} \tilde{x} .
\end{aligned}
$$

We are now ready to give the proof of Theorem 1 . Proof of Theorem 1. Let us take the candidate Lyapunov function $V(\tilde{x})=\frac{1}{2} \tilde{x}^{T} P \tilde{x}$, being $\tilde{x}=x-x_{e}$. In the flow set, $\mathcal{C}$, using its definition in (7), we get

$\langle\nabla V(\tilde{x}), f(\tilde{x}, u)\rangle=\tilde{x}^{T} P\left(A_{u}\left(\tilde{x}+x_{e}\right)+B_{u} V_{i n}\right) \leq-\eta \tilde{x}^{T} Q_{u} \tilde{x}$

In the jump set, $\mathcal{D}$, we get for all $g \in G(x)$, denoting $\tilde{x}^{+}=g-x_{e}$

$$
V\left(\tilde{x}^{+}\right)-V(\tilde{x})=\frac{1}{2}\left\{\tilde{x}^{T} P \tilde{x}-\tilde{x}^{T} P \tilde{x}\right\}=0 .
$$

Uniform global asymptotic stability is then shown applying [10, Theorem 1]. In particular, since the distance of $x$ to the attractor (10) is defined by $|x|_{\mathcal{A}}=|\tilde{x}|$, we have that [10, eq. (6)] holds from the structure of $V$ and from (18) and (19). To show practical persistent flow, we first need to build a restricted hybrid system $\mathcal{H}_{\delta, \Delta}$ by intersecting $\mathcal{C}$ and $\mathcal{D}$ with set

$$
S_{\delta, \Delta}=\{\tilde{x}:|\tilde{x}| \geq \delta \quad \text { and } \quad|\tilde{x}| \leq \Delta\}
$$


(see [10] for details). Then, notice that after each jump, from the definition of $G$ in (6) and from property (9) (in Lemma 1), we have:

$$
\tilde{x}^{T}\left(A_{q^{+}} x+B_{q^{+}} V_{i n}\right) \leq-\tilde{x}^{T} Q_{q^{+}} \tilde{x}<-\eta \tilde{x}^{T} Q_{q^{+}} \tilde{x},
$$

where we used the fact that $\eta<1$ and that $0 \notin S_{\delta, \Delta}$. Therefore, if any solution to $\mathcal{H}_{\delta, \Delta}$ performs a jump, it either jumps outside $S_{\delta, \Delta}$ (and it terminates prematurely) or, from (8), it jumps to the interior of the flow set $\mathcal{C} \cap S_{\delta, \Delta}$, Indeed, from (20) we have that $\tilde{x}$ is bounded away from zero in $S_{\delta, \Delta}$, so that, the right inequality in (21) is strict from positive definiteness of $Q_{i}, i \in \mathbb{K}$. Then all non terminating solutions must flow for some time and since $\mathcal{C} \cap S_{\delta, \Delta}$ is bounded, there is a uniform dwell-time $\rho(\delta, \Delta)$ in between each pair of consecutive jumps. This dwell-time $\rho(\delta, \Delta)$ clearly implies [10, equ. (4)] with the class $\mathcal{K}_{\infty}$ function $\gamma(j)=\rho(\delta, \Delta) j$ and $N=1$. Then, all the assumptions of [10, Theorem 1] hold and UGAS of $\mathcal{A}$ is concluded.

Below we give the proof of Theorem 2.

Proof of Theorem 2. To prove the optimality property in (14), consider any solution $\xi=(x, u)$ to $\mathcal{H}$. Then for each $(t, j) \in \operatorname{dom} x$ and denoting $t=t_{j+1}$ to simplify notation, we have from (18)

$$
\begin{aligned}
& V(\tilde{x}(t, j))-V(\tilde{x}(0,0)) \\
& =\sum_{k=0}^{j} V\left(\tilde{x}\left(t_{k+1}, k\right)\right)-V\left(\tilde{x}\left(t_{k}, k\right)\right) \\
& =\sum_{k=0}^{j} \int_{t_{k}}^{t_{k+1}}\langle\nabla V(\tilde{x}(\tau, k)), f(x(\tau, k), u(\tau, k))\rangle d \tau \\
& \leq \sum_{k=0}^{j} \int_{t_{k}}^{t_{k+1}}-\eta \tilde{x}^{T}(\tau, k) Q_{\left.u_{(} \tau, k\right)} \tilde{x}(\tau, k) d \tau \\
& \leq-\eta \sum_{k=0}^{j} \int_{t_{k}}^{t_{k+1}} \tilde{x}^{T}(\tau, k) C_{u_{(}(\tau, k)}^{T} C_{\left.u_{(}, k\right)} \tilde{x}(\tau, k) d \tau,(22),
\end{aligned}
$$

where the last inequality comes from applying (13). Now, considering $\tilde{z}(\tau, k)=C_{u(\tau, k)} \tilde{x}(t, k)$, taking the limit as $t+j \rightarrow+\infty$ and using the fact that UGAS established in Theorem 1 implies $\lim _{t+j \rightarrow+\infty} V(\tilde{x}(t, j))=0$, we get from $(22)$

$$
\eta J(\xi) \leq V(\tilde{x}(0,0))=\tilde{x}(0,0)^{T} P \tilde{x}(0,0),
$$

as to be proven.

\section{Conclusions And Future WORK}

In this article, a novel paradigm of controlling switched affine systems, specifically DC-DC converters, is provided. The control scheme, which is based on an appropriate hybrid modeling, allows managing the switching frequency with the knowledge of the state of the DCDC converters. This preliminary work finally shows the potential of considering an hybrid representation for this class of systems. For instance, we obtain a design parameter, $\eta$, that adjusts a trade-off between performance and switching frequency. We show that $\eta=1$ represents the specific controlled case obtained from other methods, as sliding-modes. An important direction for future researches would be include in this control paradigm other class of switched systems, as systems with non-Hurwitz matrices.

\section{ACKNOWLEDGMENTS}

Work supported in part by ANR under project LimICoS, contract number 12 BS03 005 01, by the iCODE institute, research project of the Idex Paris-Saclay, and by the University of Trento, grant OptHySYS.

\section{REFERENCES}

[1] G. S. Deaecto, J. C. Geromel, F.S. Garcia, and J.A. Pomilio. Switched affine systems control design with application to DCDC converters. IET control theory \& ${ }^{\prime}$ applications, 4(7):1201$1210,2010$.

[2] A.J. Forsyth and S.V. Mollov S. Modeling and control of DCDC converters. IET Power Engineering Journal, 12(5):229236, 1998.

[3] J.C. Geromel, P. Colaneri, and P. Bolzern. Dynamic output feedback control of switched linear systems. IEEE Trans. on Automatic Control, 53(3):720-733, 2008.

[4] R. Goebel, R.G. Sanfelice, and A.R. Teel. Hybrid Dynamical Systems: modeling, stability, and robustness. Princeton University Press, 2012.

[5] H. Laurentiu and F. Emilia. Robust sampled-data control of switched affine systems. IEEE Trans. on Automatic Control, 58(11):2922-2928, 2013.

[6] D. Liberzon and A.S. Morse. Basic problems in stability and design of switched systems. IEEE Control Systems Magazine, 19(5):59-70, 1999

[7] S. Mariéthoz, S. Almér, M. Bâja, A.G. Beccuti, D. Patino, A. Wernrud, J. Buisson, H. Cormerais, T. Geyer, H. Fujioka et al. Comparison of hybrid control techniques for buck and boost dc-dc converters. IEEE Trans. on Control Systems Technology, 18(5):1126-1145, 2010.

[8] L. Martínez-Salamero, G. Garcia, M. Orellana, C. Lahore, B. Estibals, C. Alonso, and C.E. Carrejo. Analysis and design of a sliding-mode strategy for start-up control and voltage regulation in a buck converter. IET Power Electronics, 6(1):52-59, 2013.

[9] C. Olalla, R. Leyva, A. El Aroudi, P. Garces, and I. Queinnec. LMI robust control design for boost PWM converters. IET Power Electronics, 3(1):75-85, 2010.

[10] C. Prieur, A. R. Teel, and L. Zaccarian. Relaxed persistent flow/jump conditions for uniform global asymptotic stability. IEEE Trans. on Automatic Control, 59(10):2766-2771, October 2014.

[11] R. G. Sanfelice, D. Copp, and P. A Nanez. A toolbox for simulation of hybrid systems in Matlab/Simulink: Hybrid equations (HyEQ) toolbox. In Hybrid Systems: Computation and Control Conference, 2013.

[12] Hebertt Sira-Ramírez and Ramón Silva-Ortigoza. Control design techniques in power electronics devices. Springer Science \& Business Media, 2006.

[13] G. Spagnuolo, G. Petrone, S.V. Araujo, C. Cecati, E. FriisMadsen, E. Gubia, D. Hissel, M. Jasinski, W. Knapp, M. Liserre, et al. Renewable energy operation and conversion schemes: A summary of discussions during the seminar on renewable energy systems. IEEE Trans. on Industrial Electronics Magazine, 4(1):38-51, 2010.

[14] C. K. Tse and K. M. Adams. Quasi-linear modeling and control of DC-DC converters. IEEE Trans. on Power Electronics, 7(2):315-323, 1992. 